\title{
Avaliação "in vivo" da composição corporal e da carcaça de caprinos - uso de ultrasonografia
}

\author{
Alfredo Teixeira ${ }^{1}$ \\ 1 Escola Superior Agrária - Centro de Investigação de Montanha. Instituto Politécnico de Bragança. Apartado 172. \\ 5301-855 Bragança Portugal. telf.: +351273303 206, Fax: +351273325405
}

RESUMO - Atendendo ao crescente interesse na produção e consumo de carne de caprinos e à cada vez maior necessidade de recorrer a metodologias rápidas que proporcionem a predição da composição corporal e qualidade da carcaça, realizamos uma revisão dos últimos trabalhos realizados com recurso à técnica dos ultra-sons. Após uma breve resenha histórica sobre a evolução do uso do método na caprinicultura, são referidos os princípios da técnica e a sua aplicação aos caprinos (operador, identificação de pontos anatómicos de medida, diferentes medidas corporais e na carcaça, tipos de aparelhos e sondas e acondicionamento animal). São posteriormente analisados os resultados das principais pesquisas recentemente efectuadas. Finalmente são discutidas as expectativas futuras para o uso da técnica de ultra-sons na avaliação in vivo de caprinos, com referência a diferentes procedimentos para a obtenção de modelos de predição.

ABSTRACT - Considering the increasing interest in the production and meat goat consumption and the necessity to appeal the rapid methodologies that provide to predict the body composition and quality of the carcass, we carry through a revision of the last works using ultrasound technology. After a brief history description about the evolution of the use of ultrasound methods in goats, the main important principles of the technique and its application in goats are refereed (operator, anatomical point's identification for measurements, different body and carcass measures, types of probes and equipment and animal handling). The most recent results of works in goats to predict carcass composition were mentioned. Finally the future expectations for the use of ultrasound technology to evaluate in vivo goats are discussed with a reference of different procedures to obtain the prediction models.

\section{Introdução}

A técnica de ultasonografia em tempo real (UTR) é uma tecnologia não invasiva que pode ser usada para predizer a composição corporal e avaliar a qualidade da composição de uma carcaça. No inicio, o uso da técnica em pequenos ruminantes não trouxe grandes expectativas a avaliar pelos trabalhos citados por Kempster et al. (1982). O custo inicial do equipamento, a pequena espessura de gordura dorsal principalmente dos caprinos em comparação com outras espécies, a presença de lã ou pelo, foram provavelmente dos principais factores que limitaram o uso da técnica de ultra-sons aos pequenos ruminantes. Na realidade os primeiros trabalhos em caprinos foram, somente, publicados em 1995 por Delfa e Stanford (Delfa et al., 1995a; e Stanford et al., 1995).

De acordo com Stoufer (2004) é evidente de que os ultrasons possuem um importante papel em ciência animal por proporcionarem avaliações de carcaças precisas, objectivas e rápidas. Ainda que o facto de ser uma técnica não evasiva seja a característica mais importante, estudos recentes em caprinos, Teixeira et al. (2008), indicam que as quantidades de músculo e gordura (subcutânea, intermuscular e total da carcaça ou do corpo) podem ser estimadas com um elevado grau de precisão.

\section{Ultrasonografia em caprinos}

À semelhança de outras espécies, hoje, de acordo com Teixeira (2008) os ultra-sons são utilizados em caprinos, principalmente, com dois objectivos: O primeiro: como ferramenta em programas de melhoramento genético para a produção de carne magra; o segundo: para a produção de carne magra através da identificação de animais que atingem os níveis óptimos de deposição de músculo e gordura para o abate, contribuindo para uma classificação comercial mais objectiva e adequada às modernas exigências do mercado. Este segundo objectivo será aquele a que 
dedicaremos especial atenção e análise no presente trabalho.

\section{Princípios do uso da técnica}

Em caprinos tal como nas demais espécies de interesse zootécnico, os aparelhos de ultrasonografia em tempo real (UTR) são os mais utilizados. Operam em branco e negro, permitindo evidenciar as interfaces mais importantes, constituídas pela gordura, músculo e osso. Estão providos dos acessórios, que permitem potenciar a sua utilização: - modo split-screen (divisão do monitor, permitindo a observação em simultâneo de duas imagens); e - modo freeze-frame (oferece a possibilidade de congelar uma imagem para estudo mais detalhado) e diferentes calipers para determinação de espessuras e áreas.

Atendendo à cada vez maior necessidade de operar em condições de campo, o mercado oferece hoje aparelhos de elevada portabilidade, em formato notebook, de peso e tamanho reduzido, mas com excelente resolução e possibilidade de ligação de todo o tipo de sondas.

Dependendo do que se pretende medir, resulta fundamental a escolha do tipo de sonda. As mais utilizadas para medir espessura de tecido adiposo ou profundidade de musculo, são as sondas lineares de aplicação externa de 7,5 e 5 MHz de frequência.

\section{Metodologia de aplicação}

Todas as avaliações de tecidos por ultra-sons estão baseadas em medidas de espessura de gordura e profundidade realizadas sobre pontos anatômicos bem precisos e definidos (Figura 1).

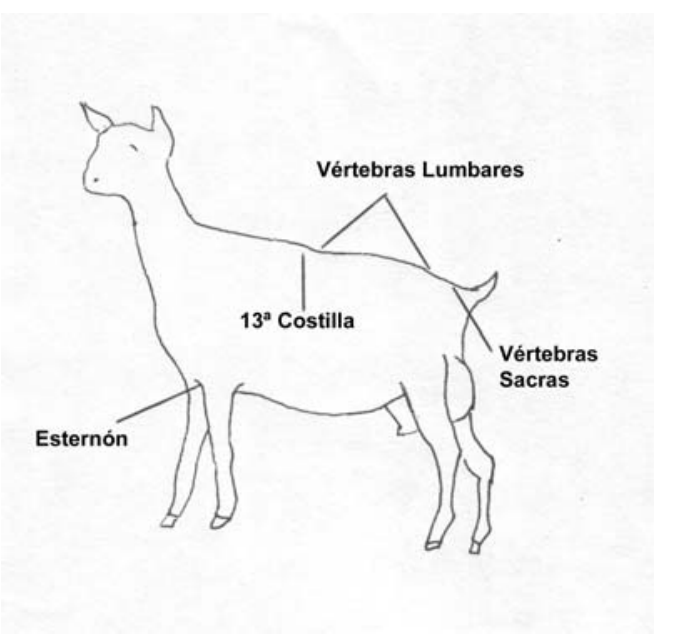

Figura 1. Pontos anatómicos de eleição para medidas em caprinos (Delfa, 2004)
Teixeira et al. (1995) demonstrou que as cabras possuem uma fina camada de deposição de gordura na região lombar em comparação com a região esternal, onde a maior espessura de camada de gordura proporciona a obtenção de medidas em melhores condições. Mais tarde, Delfa et al. (1996a) sugeriram a região do esterno como a mais adequada e precisa para avaliar a espessura de gordura subcutânea em cabras.

Assim, as principais medidas realizadas correspondem a espessura de gordura subcutânea, profundidade e área do músculo Longissimus dorsi. As suas localizações anatómicas, são: $13^{\mathrm{a}}$ costela, entre a $12^{\mathrm{a}}$ e a $13^{\mathrm{a}}$ costela, $10^{\mathrm{a}}$ costela, vértebras lombares e estenebras do esterno. As medições são realizadas com a sonda colocada perpendicularmente à coluna vertebral, quando o objectivo passa por analisar todo o olho do músculo, ou paralela a diferentes distâncias do ponto médio da coluna vertebral $(2 \mathrm{~cm}, 4 \mathrm{~cm}$ e $1 / 3)$, quando se trata de medir espessuras de gordura subcutânea (Delfa et al., 1996 ${ }^{\text {a }} 1998$ e 1999)

O acondicionamento do animal a medir, o posicionamento da sonda, o acoplamento e pressão aplicada na sonda, a experiência do operador, são factores a que deve ser dada especial atenção, com vista à obtenção de medidas precisas e isentas de erro experimental. É indispensável que o mesmo proporcione tranquilidade e relaxamento ao animal e uma posição que não colida com o normal posicionamento da sua estrutura óssea e muscular. Alguns autores usam mangas de maneio, instrumentos de sujeição especialmente desenhados, que no caso dos caprinos, pelo seu temperamento, resultam de bastante ineficácia. Nos nossos trabalhos e atendendo a que este tipo de trabalho tem de ser adaptado a uma execução rápida e algumas vezes em condições de campo, utilizamos um método de sujeição à mão, em que o técnico é ajudado por uma outra pessoa que sujeitando a cabeça do animal, sem causar stress, procura que a posição deste seja o mais natural e relaxada possível.

As medidas efectuadas na região esternal, principalmente em caprinos, podem ser efectuadas igualmente com o animal em pé ou com o animal na posição de decúbito supino em cama de endoscopia.

Muitos autores, com a finalidade de obter melhor imagem, e melhor acoplamento da sonda ao meio, tosquiam, depilam e escanhoam a região em que efectuam as suas medições. Atendendo à depreciação 
que tal procedimento provoca na pele e velo do animal e principalmente por não se adaptar às condições de trabalho num matadouro, ou mesmo a um trabalho de campo, nos nossos trabalhos não efectuamos qualquer tipo de tosquia ou corte de pelo.

Efectuamos, somente, um afastamento do velo com pente, procurando encontrar uma superfície limpa, a qual impregnamos de um agente acoplante (gel de medicina ou vaselina liquida) de modo a permitir um perfeito contacto acústico da sonda com a pele do animal, evitando a formação de bolsas de ar, de forma a permitir-nos uma visão clara (Figura 2). Na zona esternal de caprinos, por ser a maioria das vezes desprovida de lã a colocação da sonda é imediata após a impregnação da região com o gel. A pressão a aplicar na sonda sobre a superfície do animal deve ser mínima, procurando evitar a deformação dos tecidos.
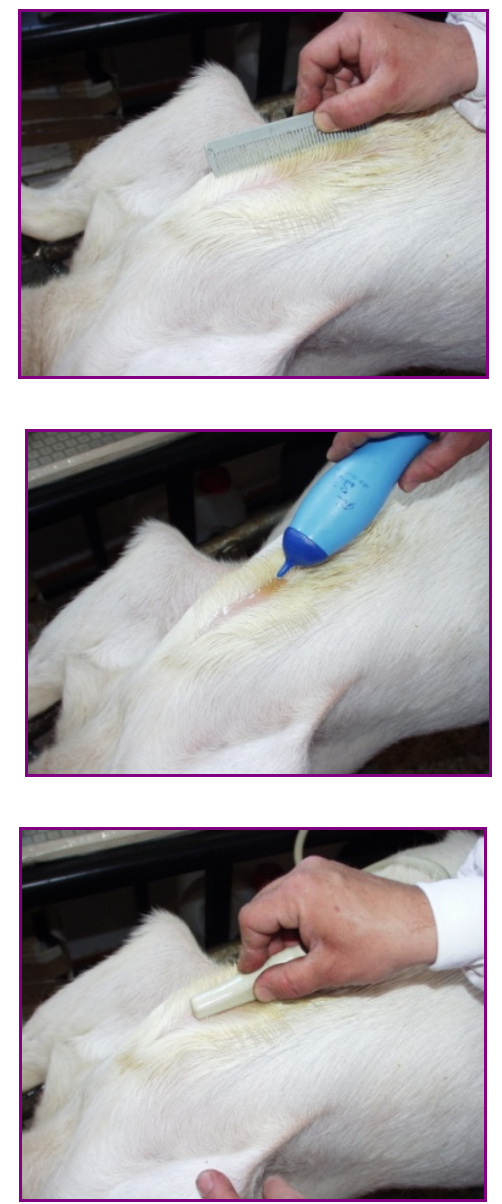

Figura 2. Método de colocação da sonda (Teixeira et al. 2008)
Nos trabalhos por nós realizados, principalmente em caprinos, em que a espessura de gordura subcutânea é mínima, e existe uma clara dificuldade em distinguir a interface entre esta camada e a pele, incluímos a espessura desta nas medidas efectuadas. A obtenção das imagens sobre as quais se efectuam as medidas, podem ser efectuadas e registadas imediatamente ou, usando um software próprio para análise de imagem pelo National Institute of Health 1.57 software (http://rsb.info.nih.gov/njh-image/ citado por Teixeira et al., 2006). Sobre a imagem e identificando com precisão os pontos anatómicos desejados, efectuam-se as medidas com a ajuda do calibrador (Figuras 3 e 4).

\section{Resultados obtidos}

Relações entre medidas tomadas in vivo e as suas homólogas na carcaça.

Em caprinos verificam-se relações altamente significativas entre as medidas realizadas com ultra-sons e as realizadas na carcaça, ambas efectuadas na região lombar $(0,70$ - 0,84; $\mathrm{P}<0,01)$. No entanto, é a nível da região esternal que foram encontradas as melhores relações entre medidas de ultra-sons e de espessura de gordura subcutânea, em cabras adultas da raça Blanca Celtibérica (Delfa et al., 1995ª 1996a , 1998; Delfa, 2004; Teixeira e tal. 2008) em cabritos da raça Blanca Celtibérica e raça Angorá (Delfa et al., 1999 e 1997', respectivamente) e em cabritos da raça Serrana (Cadavez et al., 2002 e 2007)) com coeficientes de correlação, altamente significativos, que variaram entre 0,70 e 0,98.

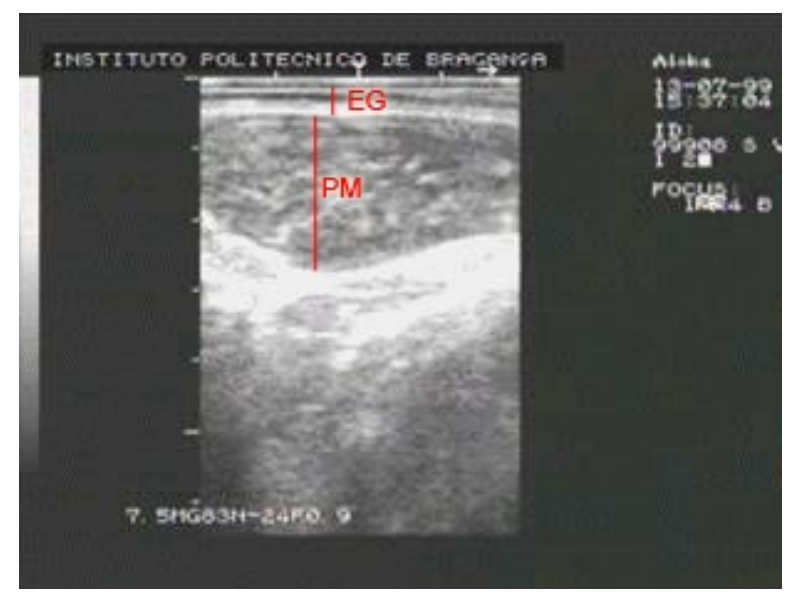

Figura 3. Imagem obtida com aparelhos de ultra-sons tipo C: PM Profundidade do músculo e EG - Espessura da gordura subcutânea (Teixeira et al., 2006) 
Relativamente à profundidade do músculo Longissimus dorsi os resultados obtidos por Delfa et al. (1998 e 1999); Delfa (2004); Cadavez et al. (2007) e Teixeira et al. (2008) encontraram uma relação altamente significativa $(0,79-0,91 ; \mathrm{P}<0,01)$ que comparada com o valor de 0,23 obtido para a raça Alpina por Stanford et al. (1995) só pode obter explicação no facto de as medidas realizadas por estes terem sido em lugar anatómico diferente, que correspondeu à primeira vértebra lombar.

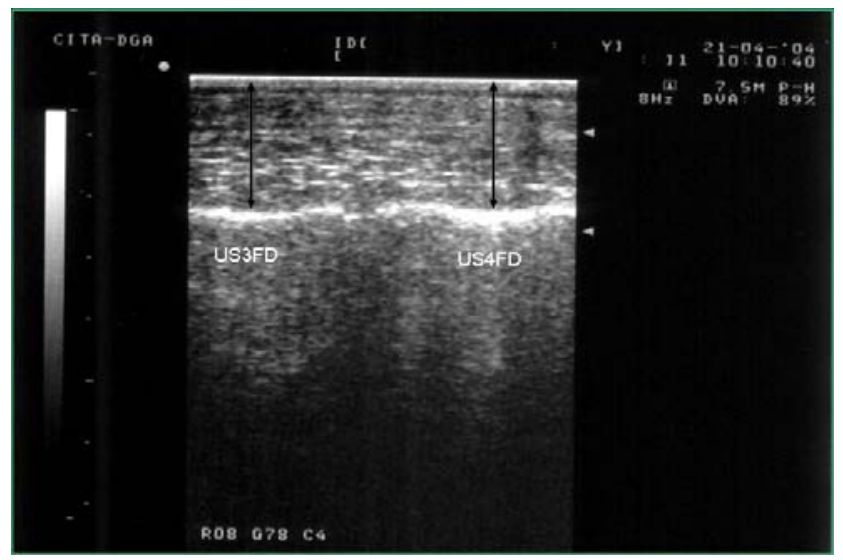

Figura 4.- Espessura da gordura subcutânea na região esternal (Teixeira et al., 2008)

\section{Predição da composição da carcaça a partir de medidas in vivo.}

As possíveis comparações entre trabalhos estão, naturalmente, dificultadas pelas diferentes condições experimentais adoptadas pelos diversos autores. Por outro lado também as comparações entre diferentes equações de predição, efectuadas com recursos aos parâmetros estatísticos que correspondem ao erro padrão residual (EPR) e coeficiente de determinação ( $R$ ou $R^{2}$ ) ficam dependentes da forma como são estimados os diferentes tecidos, se em percentagem ou em valor absoluto, conforme se pode verificar de uma análise da Tabela 1.

Em diferentes trabalhos realizados por Delfa et al. $\left(1995^{\mathrm{b}}, 1996^{\mathrm{b}}, 1997^{\mathrm{a}}\right.$ e 1998); Cadavez et al (2007) e Teixeira et al (2008) demonstraram que o peso do músculo é estimado com elevado grau de precisão pelo peso vivo associado em regressão múltipla às medidas de ultra-sons da profundidade do músculo Longissimus dorsi e espessura de gordura subcutânea a nível lombar ( $\mathrm{R}$ entre 86 e 98\%). Os mesmos autores verificaram igualmente que entre 83 e $98 \%$ da variação da gordura total da carcaça era explicada pelo mesmo conjunto de variáveis em regressão múltipla. Em algumas circunstâncias, principalmente em cabritos de peso reduzido, as medidas de ultra-sons resultaram mais eficazes as realizadas na região esternal.

Tabela 1. Diferentes estimativas de composição para caprinos.

\begin{tabular}{|c|c|c|c|c|c|}
\hline Referencias & Animais & Metodologia & Estimativa & $\mathrm{R}^{2}$ & EPR \\
\hline \multirow[t]{2}{*}{ Delfa et al. (1999) } & cabritos & Ultrasons (5) & $\begin{array}{lll}\text { Gordura } & \text { subct. } \\
\text { Interm.(g) } & +\end{array}$ & 0,98 & 57,84 \\
\hline & & Ultrasons (4) & Gordura corpo $(\mathrm{g})^{*}$ & 0,98 & 0,04 \\
\hline \multirow[t]{2}{*}{ Delfa et al. (2004) } & cabras & $\begin{array}{l}\text { Ultra-sons (2)+ Peso } \\
\text { Vivo }\end{array}$ & Gordura carcaça (g)* & 0,92 & 0,22 \\
\hline & & & Gordura do corpo (g)* & 0,94 & 2,01 \\
\hline Cadavez e tal. (2007) & cabritos & $\begin{array}{l}\text { Peso vivo + Ultra-sons } \\
\text { (2) }\end{array}$ & Musculo (g) & 0,97 & 129,8 \\
\hline \multirow[t]{3}{*}{ Teixeira et al. (2008) } & cabras & Peso vivo + Ultra-sons & Músculo (g) & 0,90 & $533,3 \mathrm{~g}$ \\
\hline & & $\begin{array}{l}\text { Ultra-sons (2) + Peso } \\
\text { vivo }\end{array}$ & $\begin{array}{l}\text { Gordura subcutânea } \\
\text { (g)* }\end{array}$ & 0,91 & 0,32 \\
\hline & & $\begin{array}{l}\text { Ultra-sons (2) + Peso } \\
\text { vivo }\end{array}$ & Gordura omental (g) & 0,91 & $685,7 \mathrm{~g}$ \\
\hline
\end{tabular}

*Após transformação logaritmica; números entre parêntesis identificam o número de variáveis independentes utilizadas; R2 coeficiente de determinação; EPR - erra padrão residual.

\section{Novos procedimentos de predição}

Em alternativa às tradicionais metodologias de tratamento estatístico, como por exemplo as diversas variantes de análise de regressão, o recurso à aplicação (c) 2008 Sociedade Brasileira de Zootecnia de redes neuronais "Artificial Neural Networks (ANN)" pode incrementar a precisão das predições, conforme demonstram alguns estudos ( em bovinos Brethour, 1994; Kim et al., 1997; Li et al., 1999; e em ovinos Cortez et al., 2006). A ANN é uma sofisticada técnica 
não linear, capaz de modelar funções complexas. A ideia é de simular a função da unidade básica do cérebro humano que é o neurónio. O número de amostras deve ser suficientemente grande, pelo menos igual ou desejavelmente maior do que o número de variáveis independentes, de forma a obter uma solução única para o sistema de redes. Podem ser estabelecidos dois modelos diferentes de ANN, tendo em conta se o objectivo final é o de obter modelos de calibração para um ou mais componentes ao mesmo tempo: ANN simples ou múltipla, respectivamente.

Em caprinos desconhece-se a utilização de tal ferramenta. Utilizando dados procedentes de estudos em cabras efectuados por Delfa (2004) e publicados por Teixeira et al. (2008), Teixeira, Peres e Dias (trabalho não publicado) utilizaram dados de 56 cabras, que foram divididos aleatoriamente em 3 grupos: 36 no grupo de treino, 10 no grupo de validação e 10 no grupo de teste ou validação externa. Foram estudados dois tipos de redes neuronais: redes de perceptron multi-camada (MLP) e redes de funções de base radial (RBF).

Os dados de peso vivo de cada animal conjuntamente com mais 12 medidas de espessura de gordura tomadas com recurso à técnica de ultrasonografia foram utilizados como variáveis independentes. Os modelos ANN de calibração e predição para as 8 variáveis dependentes (composições corporais e da carcaça em diferentes depósitos de gordura e de músculo) foram obtidos utilizando um software comercial. Para cada rede testada foi permitida uma procura do conjunto das melhores variáveis de ultrasons associadas ao peso vivo, sendo a complexidade do modelo determinada automaticamente pelo programa. A melhor rede, para cada constituinte da carcaça ou do corpo do animal, foi escolhida de forma a obter o menor erro padrão de calibração (SEC) e de validação cruzada (SECV), bem como o maior coeficiente de determinação da calibração (R2) e da validação cruzada (r2). A capacidade preditiva dos modelos de ANN estabelecidos foi ainda avaliada através do cálculo do desvio residual de predição (RPD), do erro padrão de teste (SEP) e do respectivo coeficiente de determinação (R2EV).

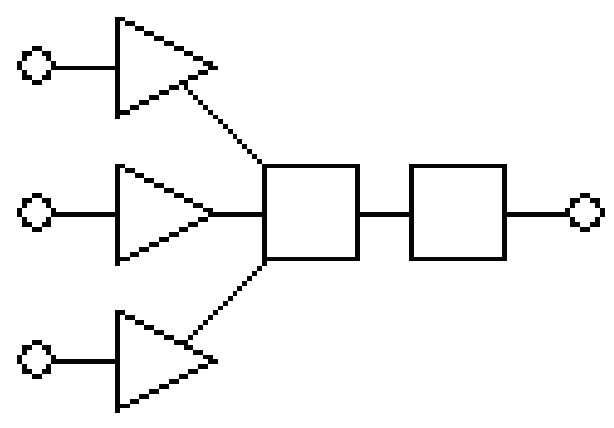

Figura 5- Rede neuronal MLP para uma variável dependente (variável gordura Omental) em função de 3 variáveis independentes (US3FD, UL5-6FD e BW).

Na figura 5 indica um exemplo de rede neuronal MLP obtida para a calibração/previsão da variável gordura Omental em função de 3 variáveis independentes (2 de medidas de espessura de gordura realizadas com ultrasons US3FD, UL5-6FD e BW peso vivo).

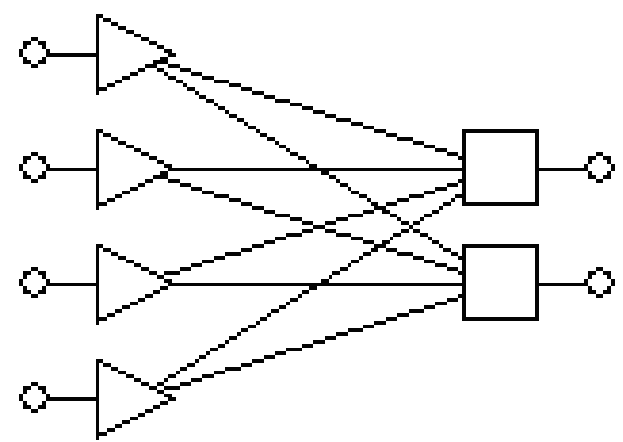

Figura 6- Rede neuronal MLP obtida para duas variáveis dependentes (variável gordura Omental e intermuscular) em função de 4 variáveis independentes (BW, US3FD, UL3-4FD e UL5-6FD).

Na Figura 6 apresenta-se um exemplo de rede neuronal MLP obtida para a calibração/previsão de duas variáveis em simultâneo (variável gordura Omental e intermuscular) em função de 4 variáveis independentes (BW peso vivo, e 3 medidas de espessura de gordura determinadas com ultrasons US3FD, UL3-4FD e UL5-6FD).

\section{Referências Bibliográficas}

Brethour, J.R. Estimating marbling score in live cattle from ultrasound images using pattern recognition and neural network procedures. J Anim. Sci. 72: 1425-1482. 1984.

CADAVEZ, V.; RODRIGUES, S.; PEREIRA, E.; DELFA, R., TEIXEIRA, A. Predición de la composicion de la canal de cabritos por ultasonografia in vivo. ITEA, v. 98A (I), p. 39-50. 2002. 
CADAVEZ, V.; RODRIGUES, S., TEIXEIRA, A. The use of ultrasonography to predict carcass composition in Kids. Agriculture - Scientific and Professional Review. Vol. 13. No. 1. 2007.

Cortez, P.; Portelinha, M,; Rodrigues, S.; Cadavez, V.; Teixiera, A. Lamb meat quality assessment by support vector machines. Neural Processing Letters, 24: 41-51. 2006

DELFA, R.; GONZÁLEZ, C.; TEIXEIRA, A. Relación entre medidas de espesor de grasa y del $\mathrm{M}$. longissimus dorsi realizadas con ultrasonidos en el animal vivo y sus homólogas tomadas en la canal de cabras adultas. ITEA, v. 16, p.651-653. 1995a.

DELFA, R.; TEIXEIRA, A.; GONZÁLEZ, C. Medidas realizadas con ultrasonidos en el animal vivo como predictoras de la composición de la canal y de los depósitos adiposos en el cuerpo de cabras adultas. ITEA, v. 16, p. 654656. 1995b.

DELFA, R.; GONZÁLEZ, C.; TEIXEIRA, A.; VIJIL, E. Ultrasonic measurements in live goats. Prediction of weight of carcass joints. Proc. 47th Annual Meeting EAAP, p. 273. 1996a.

DELFA, R.; TEIXEIRA, A.; GONZÁLEZ, C; VIJIL, E. Ultrasonic measurements for predicting carcass quality in live goats. Proc. 47th Annual Meeting EAAP, p. 272. 1996b.

DELFA, R.; CONZÁLEZ, C.; TEIXEIRA, A.; VALDERRÁBANO, J. Utilización de ultrasonidos en cabritos Angora como predictores de la calidad de sus canales. ITEA, v. 18, 733-735. 1997a.

DELFA, R.; TEIXEIRA, A.; GONZÁLEZ, C.; VALDERRÁBANO, J. Precisión de los ultrasonidos en el animal vivo para valorar diferentes medidas de la canal de cabritos de raza Blanca Celtibérica. ITEA, v. 18, p. 730-372. 1997b.

DELFA, R.; TEIXEIRA, A.; GONZÁLEZ, C. Body weight and ultrasound as predictors of carcass quality and fat partition in adult goats. Revista Portuguesa de Zootecnia, v.2, p. 1-16. 1998.
DELFA, R.; TEIXEIRA, A.; GONZÁLEZ, C.; TORRANO, L.; VALDERRÁBANO, J. Utilización de ultrasonidos en cabritos vivos de raza Blanca Celtibérica, como predictoras de la calidad se sus canales. Arch. Zootec., v. 48 (182), p. 187-196. 1999.

DELFA, R. Los ultrasonidos como predictores del reparto del tejido adiposo y de la composición tisular de la canal en cabras adultas. Tesis doctoral. Facultad de Veterinária. Universidad de Zaragoza. 2004

Kempster, A. J.; Cuthbertson, A.; Harrington, G. Carcass evaluation in livestock breeding, production and marketing. Granada: London. 306p. 1982

Kim, N-D.; Amin, V.R.; D. Wilson, D.; Rouse, G. Neural network application for classifying beef intramuscular fat percentage. Iowa State Univ. Beef and sheep Res. Rep. A. S. Leaflet R 1438, Ames. 1997

Li, J.; Tan, J.; Martz, F.A.; Heymann, H. Image texture features as indicators of beef tenderness. Meat Sci. 53: 17-22. 1999

STANFORD, K.; Mc ALLISTER, T.A.; MAC DOUGALL, M.; BAILEY, D.R.C. Use of ultrasound for the prediction of carcass characteristics in Alpine goats. Small Ruminant Research, v. 15, p. 195-201. 1995.

Stouffer, J. R. History of ultrasound in animal science. J. Ultrasound Med. 23: 577-584. 2004

Teixeira, A.; Matos, S.; Rodrigues, S.; Delfa, R.; Cadavez, V. In vivo estimation of lamb carcass composition by real-time ultrasonography. Meat Science 74: 289-295. 2006.

Teixeira, A. Basic composition: Rapid methodologies. In Handbook of Muscle Foods Analysis. Ed Leo M.L. Nollet, Fidel Toldra. Taylor \& Francis Group, LLC. In press 2008

Teixeira, A.;Joy, m., delfa, r. In vivo estimation of goat carcass composition and body fat partition by real time ultrasonography. J. Anim. Sci. Accepted April. 2008.

TEIXEIRA, a.; PERES, A.; DIAS, L. Trabalho não publicado. Comunicação pessoal. Maio de 2008. 